\title{
Adaptive Neural Output Feedback Control for Uncertain Robot Manipulators with Input Saturation
}

\author{
Rong Mei and ChengJiang Yu \\ Criminal Investigation Department, Nanjing Forest Police College, Nanjing 210023, China \\ Correspondence should be addressed to Rong Mei; meirongnuaa@163.com
}

Received 8 May 2017; Accepted 4 July 2017; Published 7 August 2017

Academic Editor: Yanan Li

Copyright (c) 2017 Rong Mei and ChengJiang Yu. This is an open access article distributed under the Creative Commons Attribution License, which permits unrestricted use, distribution, and reproduction in any medium, provided the original work is properly cited.

\begin{abstract}
This paper presents an adaptive neural output feedback control scheme for uncertain robot manipulators with input saturation using the radial basis function neural network (RBFNN) and disturbance observer. First, the RBFNN is used to approximate the system uncertainty, and the unknown approximation error of the RBFNN and the time-varying unknown external disturbance of robot manipulators are integrated as a compounded disturbance. Then, the state observer and the disturbance observer are proposed to estimate the unmeasured system state and the unknown compounded disturbance based on RBFNN. At the same time, the adaptation technique is employed to tackle the control input saturation problem. Utilizing the estimate outputs of the RBFNN, the state observer, and the disturbance observer, the adaptive neural output feedback control scheme is developed for robot manipulators using the backstepping technique. The convergence of all closed-loop signals is rigorously proved via Lyapunov analysis and the asymptotically convergent tracking error is obtained under the integrated effect of the system uncertainty, the unmeasured system state, the unknown external disturbance, and the input saturation. Finally, numerical simulation results are presented to illustrate the effectiveness of the proposed adaptive neural output feedback control scheme for uncertain robot manipulators.
\end{abstract}

\section{Introduction}

In the past decades, there have been enormous research efforts in the development of efficient control schemes for robot manipulators [1-3]. In general, robot manipulators have nonlinear characteristics and are subject to parameter perturbations and unknown disturbance $[4,5]$. Thus, the model is dynamically complex when the robot manipulator is operated in some uncertain surroundings such as underwater or in space. To achieve satisfactory control performance, a number of robust control schemes have been developed for uncertain robot manipulators, among them are universal approximator based control [6], disturbance observer based control [7], and sliding mode control [8]. To successfully complete the required tasks, it is necessary to precisely control the uncertain and nonlinear robot manipulators [9]. In [10], the trajectory and force tracking control was studied for constrained mobile manipulators with parameter uncertainty. In [11], a robust tracking control scheme was proposed for rigid robotic manipulators with uncertain dynamics. In [12], an adaptive neural network tracking control was proposed for manipulators with uncertain kinematics, dynamics, and actuator model. However, robot manipulators in operations are frequently subjected to the integrated effect of the system uncertainty, the time-varying unknown external disturbance, and the input saturation constraint. Thus, the robust tracking control schemes should be further developed for the robot manipulator to manage uncertainties, disturbances, nonlinearities, input constraints, and their coupling effects.

When dealing with system uncertainties, neural networks (NNs), being a universal approximator with learning ability, can be employed to compensate for the uncertain nonlinear dynamics in the robot manipulator [13-15]. In [16], an adaptive control was studied for robot manipulators with NN based compensation of frictional uncertainties. Tracking control was developed based on NN strategy for the robot manipulator [17]. In [18], the robust adaptive control was studied for robot manipulators using generalized fuzzy neural networks. Robust neural-fuzzy-network control was developed for the robot manipulator including actuator dynamics 
in [19]. Generally speaking, the joint position measurements can be obtained by means of encoders, which can give very accurate measurements of joint displacements. On the contrary, joint velocity measurements are more problematic due to the noise contamination nature; one solution is to use a state observer. With the state observer incorporated, the output feedback tracking controllers can be developed to make the whole closed-loop control with only position measurements [20]. To handle the unmeasured states of nonlinear systems, adaptive output feedback control methods were proposed for various nonlinear systems in [21-26]. In [27], the adaptive control law was studied for robot manipulators without velocity feedback. Neural network output feedback control was proposed for robot manipulators in [28]. In [29], the output feedback control was developed for robot manipulators based on deterministic learning method. An adaptive output feedback controller was studied for robot arms in [30]. However, the time-varying unknown disturbance and the approximation error of neural networks are usually unknown which need to be efficiently tackled in the output feedback control design.

Because robotic manipulators are subject to different types of disturbances in the changeable work environment, the control performance will be degraded. Thus, it is necessary to develop the robust control scheme to handle the unknown disturbance [31]. To fully utilize the dynamic information of the unknown external disturbance, the disturbance observer can be employed to design the robust output control scheme for the robot manipulator [32-34]. Recently, many disturbance observers have been designed to compensate for the disturbance effect. In [35], the disturbance observer based control (DOBC) was proposed for complex continuous models. The disturbance attenuation and rejection problems were investigated for a class of multi-input and multioutput (MIMO) nonlinear systems in [36]. Sliding mode control was presented for systems with mismatched uncertainties based on disturbance observer in [37]. Due to the significance of attenuating the effect of the unknown disturbance, the disturbance observer has been extensively used in robot manipulator systems [38]. The nonlinear disturbance observer was developed for robot manipulators in [39]. In [40], decentralized adaptive robust control was proposed for robot manipulators using disturbance observers. Nonlinear disturbance observer design was presented for robotic manipulators in [41]. In [42], Lyapunov-based nonlinear disturbance observer was developed for serial $n$-link robot manipulators. Robust constrained control was proposed for MIMO nonlinear systems based on disturbance observer in [43]. Although the disturbance observer technique has some practical applications in robotic manipulator control area, the disturbance observer based output feedback control strategy needs to be further investigated to enhance the antidisturbance ability by combining with the NN.

When parameter change, disturbances, or state errors become large, the required input torques will quickly reach saturation due to the needed massive control effort to maintain tracking control performance for robotic manipulators [44-52]. Under this case, the unchanged control output will destroy the stability of the closed-loop control systems.
Thus, in trajectory control of robot manipulators, the input saturation should be explicitly considered to eliminate the saturation effect [53]. The control of robot manipulators with bounded input was studied in [54]. In [55], the robust control was developed for robot manipulators with torque saturation using fuzzy logic. Fuzzy saturated output feedback tracking control was proposed for robot manipulators using a singular perturbation theory based approach in [56]. In [57], a saturated output feedback tracking control was studied for robot manipulators via fuzzy self-tuning. Disturbance observer based path tracking control was developed for robot manipulator considering torque saturation in [58]. Robust adaptive motion/force tracking control was proposed for uncertain constrained robot manipulators in [59]. In [60], a hybrid fuzzy adaptive output feedback control design was proposed for uncertain MIMO nonlinear systems with time-varying delays and input saturation. In this paper, the adaptive neural output feedback control scheme will be developed by using the RBFNN and disturbance observer for uncertain robot manipulators with input saturation based on backstepping technique. Backstepping control as an efficient control method of nonlinear systems can provide a systematic framework for the tracking control of robot manipulators. Its design flexibility has led to the robust adaptive backstepping control being extensively studied in the nonlinear control system design including the robust control design of robot manipulators [61, 62].

This work is motivated by the disturbance observer-based adaptive neural output feedback control to follow desired time-varying trajectories of robot manipulators. The state observer is designed to estimate the unmeasured state, and the disturbance observer and the RBFNN are employed to suppress the effect of the system uncertainty and the unknown external disturbance. The organization of the paper is as follows. Section 2 presents the problem descriptions of the adaptive neural output feedback control for the robot manipulator. Section 3 describes the adaptive neural output feedback control design using the sate observer, the disturbance observer, and the RBFNN based on backstepping method. Simulation studies are provided in Section 4 to demonstrate the effectiveness of the proposed disturbance observer based adaptive neural output feedback control approach, followed by some concluding remarks in Section 5 .

\section{Problem Formulation}

The dynamics of an uncertain $n$-joint robot manipulator can be described as [63]

$$
M(q) \ddot{q}+C(q, \dot{q}) \dot{q}+g(q)=u(t),
$$

where $q(t)$ is the $R^{n \times 1}$ state vector of joint angular positions; $u(t)$ is the $R^{n \times 1}$ control input vector of applied joint torques; $M(q)$ is the $R^{n \times n}$ symmetric positive-definite inertia matrix which is unknown; $C(q, \dot{q})$ is the unknown $R^{n \times n}$ matrix of Coriolis and centrifugal torques; and $g(q)$ is the unknown $R^{n \times 1}$ vector of gravitational torques. 
Defining $x=\left(q^{T}, \dot{q}^{T}\right)^{T}$ and considering (7), the uncertain robot manipulator dynamics (1) can be written as

$$
\begin{aligned}
& \dot{x}_{1}=x_{2} \\
& \dot{x}_{2}=F(x)+G_{0}\left(x_{1}\right) u(t),
\end{aligned}
$$

where $x_{1}=q, x_{2}=\dot{q}, F(x)=M(q)^{-1}(-C(q, \dot{q}) \dot{q}-g(q))$, and $G_{0}\left(x_{1}\right)=M(q)^{-1}$. Since $C(q, \dot{q})$ and $g(q)$ are unknown, $F(x)$ is also unknown which denotes the system uncertainty.

In general, the robot manipulator may suffer from the unknown external disturbance $d(t) \in R^{n \times 1}$. Here, the uncertain robot manipulator dynamics (2) can be expressed as

$$
\begin{aligned}
& \dot{x}_{1}=x_{2} \\
& \dot{x}_{2}=F(x)+G_{0}\left(x_{1}\right) u(t)+d(t) .
\end{aligned}
$$

In most practical operations, there usually exist the control input saturation $u=\operatorname{sat}(v)$, where $v$ is a desired control command. Thus, the uncertain robot manipulator dynamics (3) with input saturation can be written as

$$
\begin{aligned}
& \dot{x}_{1}=x_{2} \\
& \dot{x}_{2}=F(x)+G_{0}\left(x_{1}\right) \text { sat }(v)+d(t) .
\end{aligned}
$$

The function sat(.) represents the input saturation of robot manipulator systems and is defined as

$$
\operatorname{sat}\left(v_{i}\right)=\operatorname{sign}\left(u_{i}\right) \min \left\{u_{\max i},\left|u_{i}\right|\right\}, \quad i=1,2, \ldots, m,
$$

where $u_{\max i}$ represents the saturation level of the $i$ th input and is assumed to be known.

On the other hand, we assume the symmetric positivedefinite inertia matrix $M(q)$ with uncertainty $\Delta M(q)$. Thus, we have

$$
G_{0}\left(x_{1}\right)=G\left(x_{1}\right)+\Delta G\left(x_{1}\right),
$$

where $G\left(x_{1}\right)$ is the known nominal control gain matrix and $\Delta G\left(x_{1}\right)$ is the uncertainty of the control gain matrix.

Substituting (6) into (4) yields

$$
\begin{aligned}
& \dot{x}_{1}=x_{2} \\
& \dot{x}_{2}=F(x)+G\left(x_{1}\right) \text { sat }(v)+\Delta G\left(x_{1}\right) \text { sat }(v)+d(t) .
\end{aligned}
$$

To handle the uncertainty $F(x)$ of the robot manipulator, the following lemma is used.

Lemma 1 (see [64]). As a class of linearly parameterized NN, RBFNNs are adopted to approximate the continuous function $f(Z): R^{q} \rightarrow R$ and can be expressed as follows:

$$
f(Z)=\widehat{\Theta}^{T} \phi(Z)+\varepsilon,
$$

where $Z=\left[z_{1}, z_{2}, \ldots, z_{q}\right]^{T} \in R^{q}$ is the input vector of the $N N$, $\widehat{\Theta} \in R^{p}$ is a weight vector of the $N N, \phi(Z)=\left[\phi_{1}(Z), \phi_{2}(Z), \ldots\right.$, $\left.\phi_{p}(Z)\right]^{T} \in R^{p}$ is the basis function, and $\varepsilon$ is the approximation error of the NN. The optimal weight value $\Theta^{*}$ of RBFNN is given by

$$
\Theta^{*}=\underset{\widehat{\Theta} \in \Omega_{f}}{\arg \min _{f}}\left[\sup _{z \in S_{Z}}|\widehat{f}(Z \mid \widehat{\Theta})-f(Z)|\right],
$$

where $\Omega_{f}=\{\widehat{\Theta}:\|\widehat{\Theta}\| \leq \bar{M}\}$ is a valid field of the estimate parameter $\widehat{\Theta}, \bar{M}$ is a design parameter, and $S_{Z} \subset R^{n}$ is an allowable set of the state vector. Using the optimal weight value yields

$$
\begin{gathered}
f(Z)=\Theta^{* T} \phi(Z)+\varepsilon^{*} \\
\left|\varepsilon^{*}\right| \leq \bar{\varepsilon},
\end{gathered}
$$

where $\varepsilon^{*}$ is the optimal approximation error and $\bar{\varepsilon}>0$ is the upper bound of the approximation error.

The RBFNN is employed to approximate the system uncertainty $F(x)$ in (4) which can be expressed as $F(x)=$ $\Theta^{* T} \phi(Z)+\varepsilon$ according to (8). Considering (4), we have

$$
\begin{aligned}
\dot{x}_{1}= & x_{2} \\
\dot{x}_{2}= & G\left(x_{1}\right) \operatorname{sat}(v)+\Theta^{* T} \phi(Z)+\varepsilon^{*}+\Delta G\left(x_{1}\right) \operatorname{sat}(v) \\
& +d(t),
\end{aligned}
$$

where $Z=\left[x_{1}, x_{2}\right]^{T}$.

Due to $Z$ including $x_{2}$, it cannot directly be used in the RBFNN approximation. To solve this problem, by adding a term and subtracting a term, (11) can be rewritten as

$$
\begin{aligned}
\dot{x}_{1}= & x_{2} \\
\dot{x}_{2}= & G\left(x_{1}\right) \text { sat }(v)+\Theta^{* T} \phi(\widehat{Z})+\Theta^{* T} \phi(Z) \\
& -\Theta^{* T} \phi(\widehat{Z})+\varepsilon^{*}+\Delta G\left(x_{1}\right) \text { sat }(v)+d(t),
\end{aligned}
$$

where $\widehat{Z}=\left[\widehat{x}_{1}, \widehat{x}_{2}\right]^{T}$ and $\widehat{x}_{1}$ and $\widehat{x}_{2}$ are the estimates of $x_{1}$ and $x_{2}$, respectively.

Defining $D(t)=\Theta^{* T} \phi(Z)-\Theta^{* T} \phi(\widehat{Z})+\varepsilon^{*}+\Delta G\left(x_{1}\right) \operatorname{sat}(v)+$ $d(t)$ and the system output choosing $x_{1}$, we obtain

$$
\begin{aligned}
& \dot{x}_{1}=x_{2} \\
& \dot{x}_{2}=G\left(x_{1}\right) \text { sat }(v)+\Theta^{* T} \phi(\widehat{Z})+D(x, t) \\
& y=x_{1},
\end{aligned}
$$

where $D(t) \in R^{n \times 1}$ is called the system compounded disturbance which is unknown and $y \in R^{n \times 1}$ is the system output.

For the continuous desired joint trajectory $x_{1 d}=y_{d}$, the control objective is that the adaptive neural output feedback control input $v$ is designed to make the tracking error of each joint asymptotically convergent in the presence of system uncertainties, time-varying unknown external disturbances, 
and the input saturation. To further proceed with the adaptive neural output feedback control design, the following assumptions and lemma are needed.

Assumption 2 (see [65]). The nominal inertia matrix $M_{0}(q)$ of the robot manipulator is uniformly bounded; that is, $a_{m} I \leq$ $M_{0}(q) \leq a_{M} I$ with $a_{m}>0$ and $a_{M}>0, \forall q \in R^{n}$.

Assumption 3. There exist unknown positive constants $\theta_{0}$ and $\theta_{1}$ such that the system compounded disturbance $D$ and its time derivative $\dot{D}$ are bounded; that is, $\|D\| \leq \theta_{0}$ and $\|\dot{D}\| \leq$ $\theta_{1}$.

Assumption 4. To the desired joint trajectory $y_{d}$, there exists an unknown positive constant $\epsilon_{i}$ such that $\left\|y_{d}^{(i)}\right\| \leq \epsilon_{i}, i=$ 1,2 .

Assumption 5. For an uncertain $n$-joint robot manipulator (1) subject to the input saturation (5) and the desired reference signal $y_{d}$, there exists a feasible actual control input $v$ which can achieve the given tracking control objective. For the difference $\Delta u=\operatorname{sat}(v)-v$, without loss of generality, we assume that there exists an unknown constant $\xi \in R^{n \times 1}$ to render $\|\Delta u\| \leq \xi$.

Lemma 6 (see $[66,67])$. For bounded initial conditions, if there exists a $C^{1}$ continuous and positive-definite Lyapunov function $V(x)$ satisfying $\gamma_{1}(\|x\|) \leq V(x) \leq \gamma_{2}(\|x\|)$, such that $\dot{V}(x) \leq-\kappa V(x)+c$, where $\gamma_{1}, \gamma_{2}: R^{n} \rightarrow R$ are class $K$ functions and $\kappa$ and $c$ are positive constants, then the solution $x(t)$ is uniformly bounded.

Remark 7. For a practical robot manipulator, the input saturation should meet the physical requirement of system control. In other words, there should exist an output feedback control based on the state observer and disturbance observer that can track the given desired output of the robot manipulator in the presence of the unknown external disturbance and the input saturation for all given initial conditions. Namely, the controllability of the studied robot manipulator (1) should be satisfied under the control input saturation. To guarantee the controllability of the studied robot manipulator (1), the difference $\Delta u=\operatorname{sat}(v)-v$ between sat $(v)$ and $v$ should be bounded from a practical view. Otherwise, the controllability of the studied robot manipulator (1) cannot be satisfied. To design the adaptive output feedback control scheme, we assume that there exists an unknown constant $\xi$ to render $\|\Delta u\| \leq \rho(x) \xi$. Thus, Assumption 5 is reasonable.

Remark 8. To develop the disturbance observer that estimates the unknown system compounded disturbance, the bounded assumptions for the system compounded disturbance $D$ and its time derivative $\dot{D}$ are required. For the robot manipulator, the approximation error of the RBFNN and the external disturbance are always bounded. In addition, $\Theta^{*}$, $\phi(Z)$, and $\phi(\widehat{Z})$ are also bounded. Thus, $D$ is bounded. At the same time, from [28], we know that the approximation error $\widetilde{\omega}=\Theta^{* T} \phi(Z)-\Theta^{* T} \phi(\widehat{Z})$ is bounded. Thus, the derivatives of $\widetilde{\omega}, \varepsilon$, and $d$ are also bounded. From above analysis, Assumption 3 is reasonable due to the unknown constants $\theta_{0}$ and $\theta_{1}$.

\section{Adaptive Output Feedback Control Using Disturbance Observer}

In a practical robot manipulator, the angular velocity signal is not easily obtained and is often assumed unavailable. For the unmeasured system state $x$ and the unknown compounded disturbance $D(x, t)$ shown in (13), they cannot be directly used to design the robust adaptive control scheme for the robot manipulator based on backstepping technique. To fully utilize the dynamic information of the compounded $D(x, t)$, the disturbance observer will be proposed to estimate it. Using outputs of the RBFNN, the state observer, and the disturbance observer, the adaptive output feedback control will be designed in this section.

3.1. Design of State Observer and Disturbance Observer. To estimate the unmeasured system state, the state observer is designed as follows:

$$
\begin{aligned}
& \widehat{x}_{1}=z_{1} \\
& \widehat{x}_{2}=z_{2}+L_{2}\left(x_{1}-\hat{x}_{1}\right),
\end{aligned}
$$

where the intermediate variables $z_{1}$ and $z_{2}$ are given by

$$
\begin{aligned}
& \dot{z}_{1}=z_{2}+L_{1}\left(x_{1}-\widehat{x}_{1}\right) \\
& \dot{z}_{2}=\widehat{\Theta}^{T} \phi(\widehat{Z})+\widehat{D}+G\left(x_{1}\right) \text { sat }(v)+L_{2}\left(x_{1}-\widehat{x}_{1}\right),
\end{aligned}
$$

where $L_{1}=L_{1}^{T}>0$ and $L_{2}=L_{2}^{T}>0$ are the design parameter matrices of state observer to be determined, $\widehat{\Theta}$ is the estimate of neural network value $\Theta^{*}$, and $\widehat{D}$ is the disturbance estimate of unknown compounded disturbance $D$.

Differentiating (14) and considering (15) yield

$$
\begin{aligned}
\dot{\hat{x}}_{1}= & \widehat{x}_{2}+\left(L_{1}-L_{2}\right)\left(x_{1}-\widehat{x}_{1}\right) \\
\dot{\hat{x}}_{2}= & \widehat{\Theta}^{T} \phi(\widehat{Z})+G\left(x_{1}\right) \text { sat }(v)+\widehat{D}+L_{2}\left(x_{1}-\widehat{x}_{1}\right) \\
& +L_{2}\left(\dot{x}_{1}-\dot{\hat{x}}_{1}\right) .
\end{aligned}
$$

Invoking (13) and (16), we have

$$
\begin{aligned}
L_{2}\left(\dot{x}_{1}-\dot{\hat{x}}_{1}\right) & =L_{2}\left(x_{2}-z_{2}-L_{1}\left(x_{1}-\widehat{x}_{1}\right)\right) \\
& =L_{2}\left(x_{2}-\widehat{x}_{2}-\left(L_{1}-L_{2}\right)\left(x_{1}-\widehat{x}_{1}\right)\right) .
\end{aligned}
$$

Define $\tilde{x}_{1}=\widehat{x}_{1}-x_{1}$ and $\tilde{x}_{2}=\widehat{x}_{2}-x_{2}$. Then, considering (13), (16), and (17), we obtain

$$
\begin{aligned}
\dot{\tilde{x}}_{1}= & \tilde{x}_{2}-\left(L_{1}-L_{2}\right) \tilde{x}_{1} \\
\dot{\tilde{x}}_{2}= & \widehat{\Theta}^{T} \phi(\widehat{Z})-\Theta^{* T} \phi(\widehat{Z})+\widehat{D}-D-L_{2} \widetilde{x}_{1}-L_{2} \widetilde{x}_{2} \\
& +L_{2}\left(L_{1}-L_{2}\right) \tilde{x}_{1} \\
= & -\widetilde{\Theta}^{T} \phi(\widehat{Z})+\widetilde{D}-L_{2} \tilde{x}_{2} \\
& +\left(L_{2}\left(L_{1}-L_{2}\right)-L_{2}\right) \tilde{x}_{1},
\end{aligned}
$$

where $\widetilde{\Theta}=\Theta^{*}-\widehat{\Theta}$ and $\widetilde{D}=\widehat{D}-D$. 
The state estimate error system (18) can be written as

$$
\begin{aligned}
& \dot{\tilde{x}}=A \tilde{x}+B\left(-\widetilde{\Theta}^{T} \phi(\widehat{Z})+\widetilde{D}\right) \\
& \hat{y}=\widehat{x}_{1},
\end{aligned}
$$

where $\tilde{x}=\left[\widetilde{x}_{1}, \widetilde{x}_{2}\right]^{T}, A=\left[\begin{array}{cc}-\left(L_{1}-L_{2}\right) & I_{n \times n} \\ L_{2}\left(L_{1}-L_{2}\right)-L_{2} & -L_{2}\end{array}\right]$, and the coefficients $L_{i}, i=1,2$ are design matrix parameters and $B=$ $\left[0_{n \times n}, I_{n \times n}\right]^{T}$.

To estimate the unknown compounded disturbance $D(x, t)$ in (13), the disturbance observer is proposed as

$$
\dot{\widehat{D}}=-\widehat{D}+L_{3}\left(x_{1}-\widehat{x}_{1}\right),
$$

where $L_{3}=L_{3}^{T}>0$ is a design matrix parameter.

Considering $\widetilde{D}=\widehat{D}-D, \widetilde{x}_{1}=\widehat{x}_{1}-x_{1}$, and (20) yields

$$
\begin{aligned}
\dot{\widetilde{D}} & =-\widetilde{D}+L_{3}\left(x_{1}-\widehat{x}_{1}\right)-\dot{D}-D \\
& =-\widetilde{D}-L_{3} \widetilde{x}_{1}-\dot{D}-D .
\end{aligned}
$$

Invoking the state estimate error system (19) and the disturbance estimate error system (21), we have

$$
\begin{aligned}
\dot{\bar{X}} & =\bar{A} \widetilde{X}+\bar{B} \eta \\
\hat{y} & =\widehat{x}_{1},
\end{aligned}
$$

where $\widetilde{X}=\left[\widetilde{x}_{1}, \widetilde{x}_{2}, \widetilde{D}\right]^{T}, \bar{A}=\left[\begin{array}{ccc}-\left(L_{1}-L_{2}\right) & I_{n \times n} & 0_{n \times n} \\ L_{2}\left(L_{1}-L_{2}\right)-L_{2} & -L_{2} & I_{n^{\prime} n} \\ -L_{3} & 0_{n \times n} & -I_{n \times n}\end{array}\right]$, and the coefficients $L_{i}, i=1,2,3$, should be chosen to render that the matrix $\bar{A}$ is stable, $\bar{B}=\left[\begin{array}{ccc}0_{n \times n} & 0_{n \times n} & 0_{n \times n} \\ n_{n \times n} & I_{\times n n} \\ n_{n \times n} & 0_{n \times n} & I_{n \times n} \\ I_{n \times n}\end{array}\right]^{T}$, and $\eta=$ $\left[0,-\widetilde{\Theta}^{T} \phi(\widehat{Z}),-\dot{D}-D\right]^{T}$.

For the designed matrix $\bar{A}$, given a matrix $P=P^{T}>0$, there exists a positive-definite matrix $Q=Q^{T}>0$ such that the following matrix inequality always hold:

$$
\bar{A}^{T} P+P \bar{A} \leq-Q .
$$

Consider the Lyapunov candidate as

$$
V_{0}=\widetilde{X}^{T} P \widetilde{X}
$$

The time derivative of $V_{0}$ is

$$
\begin{aligned}
\dot{V}_{0} & =\dot{\bar{X}}^{T} P \widetilde{X}+\widetilde{X}^{T} P \dot{\bar{X}} \\
& =\widetilde{X}^{T}\left(\bar{A}^{T} P+P \bar{A}\right) \widetilde{X}+2 \widetilde{X}^{T} P \bar{B} \eta \\
& \leq-\widetilde{X}^{T} Q \widetilde{X}+2 \widetilde{X}^{T} P \bar{B} \eta .
\end{aligned}
$$

Define $P_{1}$ and $\bar{B}_{1}$ as the corresponding matrix blocks of $P$ and $\bar{B}$ for the state estimate error $\tilde{x}$ and $P_{2}$ and $\bar{B}_{2}$ as the corresponding matrix blocks of $P$ and $\bar{B}$ for the disturbance estimate error $\widetilde{D}$. Considering the following facts

$$
\begin{aligned}
& -2 \widetilde{X}^{T} P_{1} \bar{B}_{1} \widetilde{\Theta}^{T} \phi(\widehat{Z}) \leq L_{4}\|\widetilde{X}\|^{2}+\frac{1}{\lambda_{1}}\|\widetilde{\Theta}\|^{2} \\
& -2 \widetilde{D}^{T} P_{2} \bar{B}_{2}(-\dot{D}-D) \leq 2\|\widetilde{X}\|\left\|P_{2} \bar{B}_{2}\right\|(\|\dot{D}\|+\|D\|) \\
& \leq 2\left\|P_{2} \bar{B}_{2}\right\|^{2}\|\widetilde{X}\|^{2}+\|D\|^{2}+\|\dot{D}\|^{2} \\
& \leq L_{0}\|\widetilde{X}\|^{2}+\|D\|^{2}+\|\dot{D}\|^{2} \leq L_{0}\|\widetilde{X}\|^{2}+\theta_{0}^{2}+\theta_{1}^{2}
\end{aligned}
$$

we have

$$
\dot{V}_{0} \leq-\widetilde{X}^{T}\left(Q-\delta_{0} I_{3 n \times 3 n}\right) \widetilde{X}+\frac{1}{\lambda_{1}}\|\widetilde{\Theta}\|^{2}+\theta_{0}^{2}+\theta_{1}^{2},
$$

where $L_{0}=2\|P \bar{B}\|^{2}, L_{4}=\lambda_{1}\|P \bar{B}\|^{2} \mu^{2}, \delta_{0}=L_{0}+L_{4},\|\phi(\widehat{Z})\| \leq$ $\mu$, and $\lambda_{1}>0$ is a design constant.

\subsection{Design of Output Feedback Using Backstepping Method.} Up to now, the state observer and the disturbance observer have been proposed for the uncertain robot manipulator using the RBFNN. Next, the adaptive neural output feedback control based on the developed disturbance observer will be proposed for uncertain robot manipulators with input saturation using the backstepping method and adaptation technique. The detailed design procedure is described as follows.

Step 1. To develop the adaptive neural output feedback control scheme, we define

$$
\begin{aligned}
& e_{1}=\widehat{x}_{1}-y_{d} \\
& e_{2}=\widehat{x}_{2}-\alpha_{1}-\dot{y}_{d},
\end{aligned}
$$

where $\alpha_{1}$ is a virtual control law which will be designed.

Considering (16) and differentiating $e_{1}$ with respect to time yield

$$
\dot{e}_{1}=\dot{\hat{x}}_{1}-\dot{y}_{d}=\widehat{x}_{2}+\left(L_{1}-L_{2}\right)\left(x_{1}-\widehat{x}_{1}\right)-\dot{y}_{d} .
$$

Considering (29), we obtain

$$
\dot{e}_{1}=e_{2}+\alpha_{1}+\left(L_{1}-L_{2}\right)\left(x_{1}-\widehat{x}_{1}\right) .
$$

The virtual control law $\alpha_{1}$ is designed as

$$
\alpha_{1}=-K_{1} e_{1}-\left(L_{1}-L_{2}\right)\left(x_{1}-\widehat{x}_{1}\right),
$$

where $K_{1}=K_{1}^{T}>0$ is a design parameter.

Substituting (32) into (31), we have

$$
\dot{e}_{1}=-K_{1} e_{1}+e_{2} .
$$

Consider the Lyapunov function candidate as

$$
V_{1}=\frac{1}{2} e_{1}^{T} e_{1}
$$

Invoking (33), the time derivative of $V_{1}$ is given by

$$
\dot{V}_{1}=-e_{1}^{T} K_{1} e_{1}+e_{1}^{T} e_{2} .
$$


Step 2. Considering (16) and (29) and differentiating $e_{2}$ with respect to time yield

$$
\begin{aligned}
\dot{e}_{2}= & \dot{\hat{x}}_{2}-\dot{\alpha}_{1}-\ddot{y}_{d} \\
= & \widehat{\Theta}^{T} \phi(\widehat{Z})+G\left(x_{1}\right) \operatorname{sat}(v)+\widehat{D}+L_{2}\left(x_{1}-\widehat{x}_{1}\right) \\
& +L_{2}\left(\dot{x}_{1}-\dot{\hat{x}}_{1}\right)-\dot{\alpha}_{1}-\ddot{y}_{d},
\end{aligned}
$$

where $\dot{\alpha}_{1}=-K_{1} \dot{e}_{1}-\left(L_{1}-L_{2}\right)\left(x_{2}-\widehat{x}_{2}-\left(L_{1}-L_{2}\right)\left(x_{1}-\widehat{x}_{1}\right)\right)$.

Defining $\varsigma=-K_{1} \dot{e}_{1}+\left(L_{1}-L_{2}\right)\left(L_{1}-L_{2}\right)\left(x_{1}-\widehat{x}_{1}\right)$, we have

$$
\dot{\alpha}_{1}=\varsigma+\left(L_{1}-L_{2}\right) \tilde{x}_{2} .
$$

Invoking (17) and (37), (36) can be written as

$$
\begin{aligned}
\dot{e}_{2}= & \widehat{\Theta}^{T} \phi(\widehat{Z})+G\left(x_{1}\right) \text { sat }(v)+\widehat{D}+L_{2}\left(x_{1}-\widehat{x}_{1}\right) \\
& +L_{2}\left(x_{2}-\widehat{x}_{2}\right)-L_{2}\left(L_{1}-L_{2}\right)\left(x_{1}-\widehat{x}_{1}\right)-\varsigma \\
& -\left(L_{1}-L_{2}\right) \tilde{x}_{2}-\ddot{y}_{d} .
\end{aligned}
$$

Considering $\Delta u=\operatorname{sat}(v)-v$ and $\tilde{x}_{2}=\widehat{x}_{2}-x_{2}$, (38) can be expressed as

$$
\begin{aligned}
\dot{e}_{2}= & \widehat{\Theta}^{T} \phi(\widehat{Z})+G\left(x_{1}\right) v+G\left(x_{1}\right) \Delta u+\widehat{D} \\
& +L_{2}\left(x_{1}-\widehat{x}_{1}\right)-L_{2} \tilde{x}_{2}-L_{2}\left(L_{1}-L_{2}\right)\left(x_{1}-\widehat{x}_{1}\right) \\
& -\varsigma-\left(L_{1}-L_{2}\right) \tilde{x}_{2}-\ddot{y}_{d} .
\end{aligned}
$$

Utilizing the outputs of the disturbance observer and the RBFNN, the adaptive neural output feedback control law $v$ is designed as

$$
\begin{aligned}
v= & -G(x)^{-1} \\
& \cdot\left(K_{2} e_{2}+\widehat{\Theta}^{T} \phi(\widehat{Z})+\widehat{D}-\varsigma-\ddot{y}_{d}+e_{1}+v_{0}\right) \\
v_{0} & =L_{2}\left(x_{1}-\widehat{x}_{1}\right)-L_{2}\left(L_{1}-L_{2}\right)\left(x_{1}-\widehat{x}_{1}\right) \\
& +\operatorname{sign}\left(e_{2}\right) g\left(x_{1}\right) \rho(\widehat{x}) \widehat{\xi},
\end{aligned}
$$

where $K_{2}=K_{2}^{T}>0, \operatorname{sign}\left(e_{2}\right)=\operatorname{diag}\left\{\operatorname{sign}\left(e_{2 i}\right)\right\}$, with $\operatorname{sign}(\cdot)$ denoting the sign function, $g\left(x_{1}\right)=\left[\left\|g_{1}\left(x_{1}\right)\right\|,\left\|g_{2}\left(x_{1}\right)\right\|\right.$, $\left.\ldots,\left\|g_{n}\left(x_{1}\right)\right\|\right]^{T}$, with $g_{i}\left(x_{1}\right)$ denoting the $i$ th row of input matrix $G\left(x_{1}\right)$, and $\widehat{\xi}$ is an estimate of unknown constant $\xi$.

Substituting (40) into (39), we obtain

$$
\begin{aligned}
\dot{e}_{2}= & -K_{2} e_{2}-e_{1}-\operatorname{sign}\left(e_{2}\right) g\left(x_{1}\right) \rho(\widehat{x}) \widehat{\xi}+G\left(x_{1}\right) \Delta u \\
& -L_{2} \tilde{x}_{2}-\left(L_{1}-L_{2}\right) \tilde{x}_{2} \\
= & -K_{2} e_{2}-e_{1}-\operatorname{sign}\left(e_{2}\right) g\left(x_{1}\right) \rho(\widehat{x}) \widehat{\xi}+G\left(x_{1}\right) \Delta u \\
& -L_{1} \tilde{x}_{2} .
\end{aligned}
$$

In accordance with (41), we have

$$
\begin{aligned}
e_{2}^{T} \dot{e}_{2}= & -e_{2}^{T} K_{2} e_{2}-e_{2}^{T} e_{1}-e_{2}^{T} \operatorname{sign}\left(e_{2}\right) g\left(x_{1}\right) \rho(\widehat{x}) \hat{\xi} \\
& +e_{2}^{T} G\left(x_{1}\right) \Delta u-e_{2}^{T} L_{1} \tilde{x}_{2} .
\end{aligned}
$$

Considering $\|\Delta u\| \leq \rho(x) \xi$, we obtain

$$
\begin{aligned}
& e_{2}^{T} \operatorname{sign}\left(e_{2}\right) g\left(x_{1}\right) \rho(\widehat{x}) \widehat{\xi}=\sum_{i=1}^{n}\left|e_{2 i}\right|\left\|g_{i}\left(x_{1}\right)\right\| \rho(\widehat{x}) \widehat{\xi} \\
& e_{2}^{T} G\left(x_{1}\right) \Delta u \\
& \quad=e_{2}^{T}\left[g_{1}\left(x_{1}\right) \Delta u, g_{2}\left(x_{1}\right) \Delta u, \ldots, g_{n}\left(x_{1}\right) \Delta u\right]^{T} \\
& \quad \leq \sum_{i=1}^{n}\left|e_{2 i}\right|\left\|g_{i}\left(x_{1}\right)\right\| \rho(x) \xi .
\end{aligned}
$$

Substituting (43) into (42) yields

$$
\begin{aligned}
e_{2}^{T} \dot{e}_{2} \leq & -e_{2}^{T} K_{2} e_{2}-e_{2}^{T} e_{1}-\sum_{i=1}^{n}\left|e_{2 i}\right|\left\|g_{i}\left(x_{1}\right)\right\| \rho(\widehat{x}) \widehat{\xi} \\
& +\sum_{i=1}^{n}\left|e_{2 i}\right|\left\|g_{i}\left(x_{1}\right)\right\| \rho(x) \xi-e_{2}^{T} L_{1} \tilde{x}_{2} \\
= & -e_{2}^{T} K_{2} e_{2}-e_{2}^{T} e_{1}-\sum_{i=1}^{n}\left|e_{2 i}\right|\left\|g_{i}\left(x_{1}\right)\right\| \rho(\widehat{x}) \tilde{\xi} \\
& -e_{2}^{T} L_{1} \tilde{x}_{2}+\sum_{i=1}^{n}\left|e_{2 i}\right|\left\|g_{i}\left(x_{1}\right)\right\|(\rho(x)-\rho(\widehat{x})) \xi,
\end{aligned}
$$

where $\widetilde{\xi}=\widehat{\xi}-\xi$.

Considering Assumption 2, we can obtain $\left\|g_{i}\left(x_{1}\right)\right\| \leq \beta_{0}$ with $\beta_{0}>0$. Invoking Assumption 5, we have

$$
\begin{aligned}
& \sum_{i=1}^{n}\left|e_{2 i}\right|\left\|g_{i}\left(x_{1}\right)\right\|(\rho(x)-\rho(\widehat{x})) \xi \\
& \leq l \beta_{0}\|\xi\|\|\tilde{x}\| \sum_{i=1}^{n}\left|e_{2 i}\right| \\
& \quad \leq \frac{1}{2}\left(l \beta_{0}\|\xi\|\right)^{2}\left\|e_{2}\right\|^{2}+\frac{1}{2}\|\widetilde{X}\|^{2} .
\end{aligned}
$$

Consider the Lyapunov function candidate

$$
V_{2}=\frac{1}{2} e_{2}^{T} e_{2}+\frac{1}{2 \gamma} \widetilde{\xi}^{T} \widetilde{\xi}+\frac{1}{2} \operatorname{tr}\left(\widetilde{\Theta}^{T} \Lambda^{-1} \widetilde{\Theta}\right),
$$

where $\gamma>0$ and $\Lambda=\Lambda^{T}>0$ are design parameters.

Invoking (44), the time derivative of $V_{2}$ is

$$
\begin{aligned}
\dot{V}_{2}= & e_{2}^{T} \dot{e}_{2}+\frac{1}{\gamma} \widetilde{\xi}^{T} \dot{\widetilde{\xi}}+\operatorname{tr}\left(\widetilde{\Theta}^{T} \Lambda^{-1} \dot{\widetilde{\Theta}}\right) \\
\leq & -e_{2}^{T} K_{2} e_{2}-e_{2}^{T} e_{1}-\sum_{i=1}^{n}\left|e_{2 i}\right|\left\|g_{i}\left(x_{1}\right)\right\| \rho(\widehat{x}) \widetilde{\xi} \\
& +\frac{1}{2}\left(l \beta_{0}\|\xi\|\right)^{2}\left\|e_{2}\right\|^{2}+\frac{1}{2}\|\widetilde{X}\|^{2}-e_{2}^{T} L_{1} \tilde{x}_{2} \\
& +\frac{1}{\gamma} \widetilde{\xi}^{T} \dot{\tilde{\xi}}-\operatorname{tr}\left(\widetilde{\Theta}^{T} \Lambda^{-1} \dot{\tilde{\Theta}}\right) .
\end{aligned}
$$


The parameter adaptation laws of $\widehat{\xi}$ and $\widehat{\Theta}$ are designed as

$$
\begin{aligned}
\dot{\vec{\xi}} & =\gamma\left(\left|e_{2 i}\right|\left\|g_{i}\left(x_{1}\right)\right\| \rho^{T}(\widehat{x})-\sigma_{1} \widehat{\xi}\right) \\
\dot{\widehat{\Theta}} & =\Lambda\left(\phi(\widehat{Z}) e_{2}+\sigma_{2} \widehat{\Theta}\right),
\end{aligned}
$$

where $\sigma_{1}>0$ and $\sigma_{2}>0$ are the design parameters.

Substituting (48) into (47) and considering $\tilde{\xi}=\hat{\xi}-\xi$ and $\widetilde{\Theta}=\Theta^{*}-\widehat{\Theta}$, we obtain

$$
\begin{aligned}
\dot{V}_{2} \leq & -e_{2}^{T} K_{2} e_{2}-e_{2}^{T} e_{1}-e_{2}^{T} L_{1} \widetilde{x}_{2}-\sigma_{1} \widetilde{\xi} \widehat{\xi}-e_{2}^{T} \widetilde{\Theta}^{T} \phi(\widehat{Z}) \\
& -\sigma_{2} \operatorname{tr}\left(\widetilde{\Theta}^{T} \widehat{\Theta}\right)+\frac{1}{2}\left(l \beta_{0}\|\xi\|\right)^{2}\left\|e_{2}\right\|^{2}+\frac{1}{2}\|\widetilde{X}\|^{2} .
\end{aligned}
$$

Considering the following facts

$$
\begin{aligned}
2 \widetilde{\xi} \widetilde{\xi} & \geq \widetilde{\xi}^{2}-\xi^{2} \\
-2 e_{2}^{T} L_{1} \widetilde{x}_{2} & \leq\left\|L_{1}\right\|^{2}\left\|e_{2}\right\|^{2}+\|\widetilde{X}\|^{2} \\
-2 e_{2}^{T} \widetilde{\Theta}^{T} \phi(\widehat{Z}) & \leq \lambda_{2} \mu^{2}\left\|e_{2}\right\|^{2}+\frac{1}{\lambda_{2}}\|\widetilde{\Theta}\|^{2} \\
2 \operatorname{tr}\left(\widetilde{\Theta}^{T} \widehat{\Theta}\right) & =\|\widetilde{\Theta}\|^{2}+\|\widehat{\Theta}\|-\left\|\Theta^{*}\right\| \geq\|\widetilde{\Theta}\|^{2}-\left\|\Theta^{*}\right\|
\end{aligned}
$$

we have

$$
\begin{aligned}
\dot{V}_{2} \leq & -e_{2}^{T}\left(K_{2}-\delta_{1} I_{3 n \times 3 n}\right) e_{2}-\frac{\sigma_{1}}{2} \tilde{\xi}^{2} \\
& -\left(\frac{\sigma_{2}}{2}-\frac{1}{2 \lambda_{2}}\right)\|\widetilde{\Theta}\|^{2}-e_{2}^{T} e_{1}+\|\widetilde{X}\|^{2}+\frac{\sigma_{1}}{2} \xi^{2} \\
& +\frac{1}{2}\left\|\Theta^{*}\right\|^{2},
\end{aligned}
$$

where $\lambda_{2}>0$ is a design parameter and $\delta_{1}=(1 / 2)\left\|L_{1}\right\|^{2}+$ $(1 / 2) \lambda_{2} \mu^{2}+(1 / 2)\left(l \beta_{0}\|\xi\|\right)^{2}$.

To analyze the convergence of the whole closed-loop system, the Lyapunov function candidate is considered as

$$
V=\sum_{i=0}^{2} V_{i}
$$

Considering (27), (35), and (51) yields

$$
\begin{aligned}
\dot{V} \leq & -\widetilde{X}^{T}\left(Q-\delta_{0} I_{3 n \times 3 n}\right) \widetilde{X}+\frac{1}{\lambda_{1}}\|\widetilde{\Theta}\|^{2}+\theta_{0}^{2}+\theta_{1}^{2} \\
& -e_{1}^{T} K_{1} e_{1}-e_{2}^{T}\left(K_{2}-\delta_{1} I_{3 n \times 3 n}\right) e_{2}-\frac{\sigma_{1}}{2} \widetilde{\xi}^{2} \\
& -\left(\frac{\sigma_{2}}{2}-\frac{1}{2 \lambda_{2}}\right)\|\widetilde{\Theta}\|^{2}+\|\widetilde{X}\|^{2}+\frac{\sigma_{1}}{2} \xi^{2}+\frac{1}{2}\left\|\Theta^{*}\right\|^{2} \\
= & -\widetilde{X}^{T}\left(Q-\left(\delta_{0}+1\right) I_{3 n \times 3 n}\right) \widetilde{X}-e_{1}^{T} K_{1} e_{1} \\
& -e_{2}^{T}\left(K_{2}-\delta_{1} I_{3 n \times 3 n}\right) e_{2}-\frac{\sigma_{1}}{2} \widetilde{\xi}^{2} \\
& -\left(\frac{\sigma_{2}}{2}-\frac{1}{\lambda_{1}}-\frac{1}{2 \lambda_{2}}\right)\|\widetilde{\Theta}\|^{2}+\frac{\sigma_{1}}{2} \xi^{2}+\frac{1}{2}\left\|\Theta^{*}\right\|^{2} \\
& +\theta_{0}^{2}+\theta_{1}^{2} \leq-\kappa V+C,
\end{aligned}
$$

where

$\kappa$

$$
\begin{aligned}
& =\min \left(\begin{array}{c}
\lambda_{\min }\left(\mathrm{Q}-\left(\delta_{0}+1\right) I_{3 n \times 3 n}\right), 2 \lambda_{\min }\left(K_{1}\right), \\
\left.2 \lambda_{\min }\left(K_{2}-\delta_{1} I_{3 n \times 3 n}\right), \sigma_{1}, \frac{2\left(\sigma_{2} / 2-1 / \lambda_{1}-1 / 2 \lambda_{2}\right)}{\lambda_{\max }(\Lambda)}\right)
\end{array}\right) \\
& >0 \\
& C=\frac{\sigma_{1}}{2} \xi^{2}+\frac{1}{2}\left\|\Theta^{*}\right\|^{2}+\theta_{0}^{2}+\theta_{1}^{2}>0 .
\end{aligned}
$$

Integration of (53) yields

$$
0 \leq V \leq \frac{C}{\kappa}+\left(V(0)-\frac{C}{\kappa}\right) e^{-\kappa t} .
$$

The disturbance observer based adaptive neural output feedback control design procedure of the uncertain robot manipulator can be summarized in the following theorem, which includes the result of disturbance observer based adaptive neural output feedback control with unknown system uncertainty, time-varying external disturbance, and input saturation.

Theorem 9. Consider the robot manipulator system (1) with unknown time-varying external disturbance. The state observer (14) with (15) is designed for the robot manipulator. The unknown compounded disturbance is estimated using the disturbance observer (20). Using the outputs of the state observer, the disturbance observer, and the RBFNN, the adaptive neural output feedback control for the robot manipulator is designed as (40), with the parameter updated laws (48). Then, all closed-loop system signals are semiglobally uniformly bounded under the proposed disturbance observer based adaptive neural output feedback control scheme.

Proof. According to (55), we can obtain that $V$ is exponentially convergent, and $\lim _{t \rightarrow \infty} V=C / \kappa$. Thus, all signals of the closed-loop system are uniformly ultimately bounded. Due to bounded $e_{1}$ and $\widetilde{x}_{1}$, we can know that the tracking error $y-y_{d}$ is bounded under the proposed adaptive output feedback control scheme from (28) and (55). This concludes the proof.

\section{Simulation Results}

To illustrate the effectiveness of the developed adaptive neural output feedback control scheme based on the disturbance observer, numerical simulation results are presented for the robot manipulator. The dynamic equations of a two-link robotic manipulator can be written as [63]

$$
\begin{aligned}
& {\left[\begin{array}{ll}
a_{11}\left(q_{2}\right) & a_{12}\left(q_{2}\right) \\
a_{12}\left(q_{2}\right) & a_{22}\left(q_{2}\right)
\end{array}\right]\left[\begin{array}{l}
\ddot{q}_{1} \\
\ddot{q}_{2}
\end{array}\right]} \\
& =\left[\begin{array}{c}
\beta_{12} \dot{q}_{1}^{2}+2 \beta_{12}\left(q_{2}\right) \dot{q}_{1} \dot{q}_{2} \\
-\beta_{12}\left(q_{2}\right) \dot{q}_{2}^{2}
\end{array}\right]+\left[\begin{array}{l}
\gamma_{01}\left(q_{1}, q_{2}\right) g \\
\gamma_{02}\left(q_{1}, q_{2}\right) g
\end{array}\right] \\
& +\left[\begin{array}{l}
u_{1} \\
u_{2}
\end{array}\right]+\left[\begin{array}{l}
d_{1} \\
d_{2}
\end{array}\right]
\end{aligned}
$$


TABLE 1: Parameters of the two-link robotic manipulator.

\begin{tabular}{lc}
\hline Parameter & Value \\
\hline$r_{1}$ & $1 \mathrm{~m}$ \\
$r_{2}$ & $0.8 \mathrm{~m}$ \\
$J_{1}$ & $5 \mathrm{~kg} \cdot \mathrm{m}$ \\
$J_{2}$ & $5 \mathrm{~kg} \cdot \mathrm{m}$ \\
$m_{1}$ & $0.5 \mathrm{~kg}$ \\
$m_{2}$ & $1.5 \mathrm{~kg}$ \\
\hline
\end{tabular}

where $a_{11}\left(q_{2}\right)=\left(m_{1}+m_{2}\right) r_{1}^{2}+m_{2} r_{2}^{2}+2 m_{2} r_{1} r_{2} \cos \left(q_{2}\right)+$ $J_{1}, a_{12}\left(q_{2}\right)=m_{2} r_{2}^{2}+m_{2} r_{1} r_{2} \cos \left(q_{2}\right), a_{22}=m_{2} r_{2}^{2}+J_{2}$, $\beta_{12}=m_{2} r_{1} r_{2} \sin \left(q_{2}\right), \gamma_{01}\left(q_{1}, q_{2}\right)=-\left(\left(m_{1}+m_{2}\right) r_{1} \cos \left(q_{2}\right)+\right.$ $\left.m_{2} r_{2} \cos \left(q_{1}+q_{2}\right)\right), \gamma_{02}\left(q_{1}, q_{2}\right)=-m_{2} r_{2} \cos \left(q_{1}+q_{2}\right)$, and $d_{i}(i=$ $1,2)$ represent the unknown time-varying disturbances of the robot manipulator system. We assume that $M_{0}(q)=$

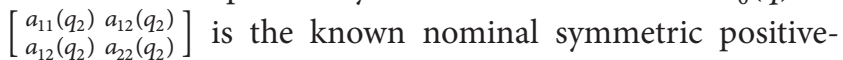
definite inertia matrix and $\Delta M(q)=0.15 M_{0}(q)$ is the uncertainty of the symmetric positive-definite inertia matrix. All parameters of the two-link robotic manipulator are given in Table 1 [63].

In the simulation study, the state observer is designed as (14) and (15). The compounded disturbance is estimated using the disturbance observer which is proposed as (20). Using the output of the state observer, the disturbance observer, and the RBFNN, the adaptive neural output feedback control of the robot manipulator is designed as (40) and the parameter updated laws are chosen as (48).

Since the sign function term $\operatorname{sign}\left(e_{2 i}\right)$ in the adaptive neural output feedback control design will introduce a highfrequency oscillation into the system that can excite the unmodeled dynamics, to avoid chattering, function $\operatorname{sign}\left(e_{2 i}\right)$ is replaced with sat $\left(e_{2 i} / \tau_{i}\right)$ in the simulation, where $\tau_{i}$ is the boundary layer width and sat $\left(e_{2 i} / \tau_{i}\right)$ is given by [68]

$$
\operatorname{sat}\left(\frac{e_{2 i}}{\tau}\right)= \begin{cases}\frac{e_{2 i}}{\tau}, & \left|\frac{e_{2 i}}{\tau}\right| \leq 1 \\ \operatorname{sign}\left(\frac{e_{2 i}}{\tau_{i}}\right), & \left|\frac{e_{2 i}}{\tau_{i}}\right|>1 .\end{cases}
$$

The initial condition of the updated parameters is chosen as $\widehat{W}=\operatorname{diag}\{0.1\}$ and $\widehat{\theta}=[0,0]^{T}$. All design parameters of the disturbance observer based adaptive neural output feedback control are chosen as $L_{1}=\operatorname{diag}\{200\}, L_{2}=\operatorname{diag}\{300\}, L_{3}=$ $\operatorname{diag}\{100\} \gamma=0.2, \sigma_{1}=0.2, \Lambda=\operatorname{diag}\{2\}, \sigma_{1}=0.5, \tau_{i}=0.2$, $K_{1}=\operatorname{diag}\{120\}$, and $L_{2}=\operatorname{diag}\{135\}$.

To illustrate the effectiveness of the proposed disturbance observer based adaptive neural output feedback control scheme, the tracking control simulation results are presented for the time-varying desired tracking signals.

The desired trajectories are taken as $x_{11 d}=0.6 \cos (0.6 t)+$ $1.5 \cos (1.5 t)$ and $x_{12 d}=0.4 \cos (0.4 t)+0.6 \cos (1.2 t)$ and the unknown disturbances are assumed as $D_{1}=2 \sin (t)$ and $D_{2}=1.2 \cos (1.5 t)$. The initial states are chosen as $x_{1}=$ $[0.5,-1]^{T}$ and $x_{2}=[0.1,0.1]^{T}$.

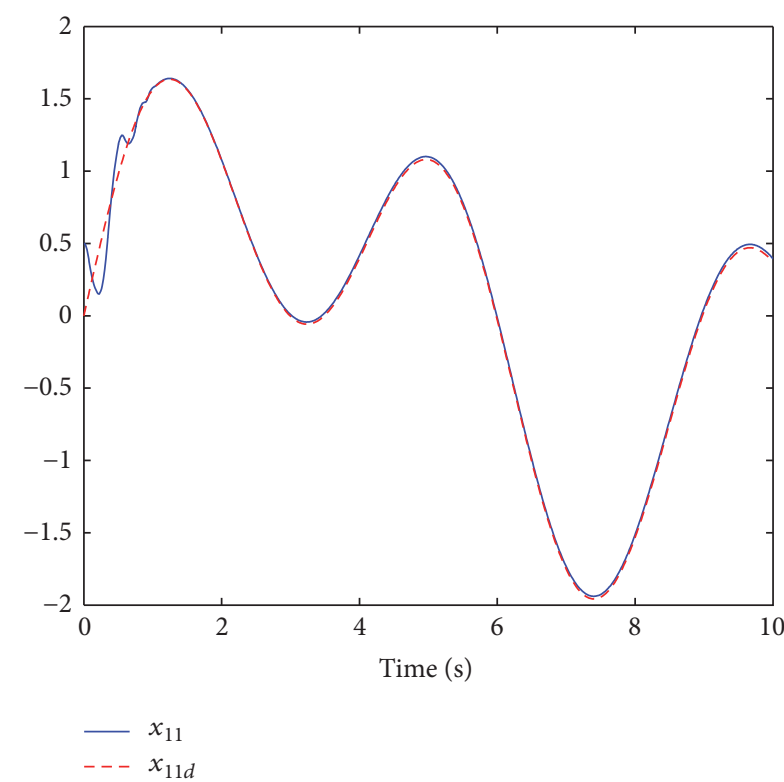

FIGURE 1: Tracking result of $x_{11}$ in response to $x_{11 d}$.

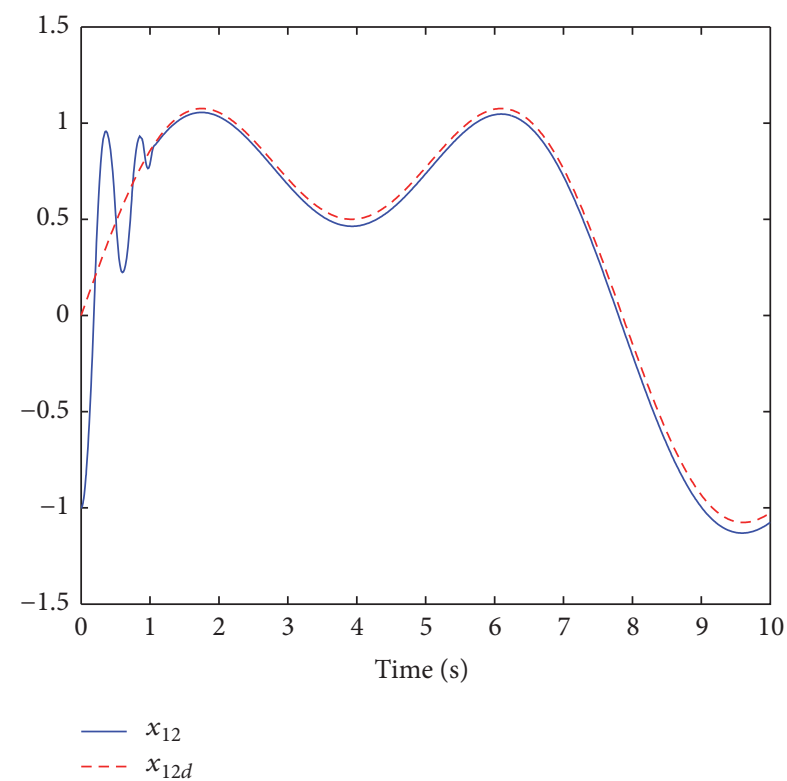

FIGURE 2: Tracking result of $x_{12}$ in response to $x_{12 d}$.

The tracking control results of the two-link robotic manipulator are shown in Figures 1, 2, and 3 under the proposed adaptive neural output feedback control scheme. From Figures 1-3, for the time-varying desired trajectories, we note that $x_{11}$ and $x_{12}$ can track the corresponding desired signals $x_{11 d}$ and $x_{12 d}$ with a small tracking error under the integrated system uncertainty, consisting of the unmeasured system state, the unknown external disturbance, and the input saturation. At the same time, the state estimate results of two joints are given in Figures 4-7. In accordance with the simulation results of state observer, we can see that the state 


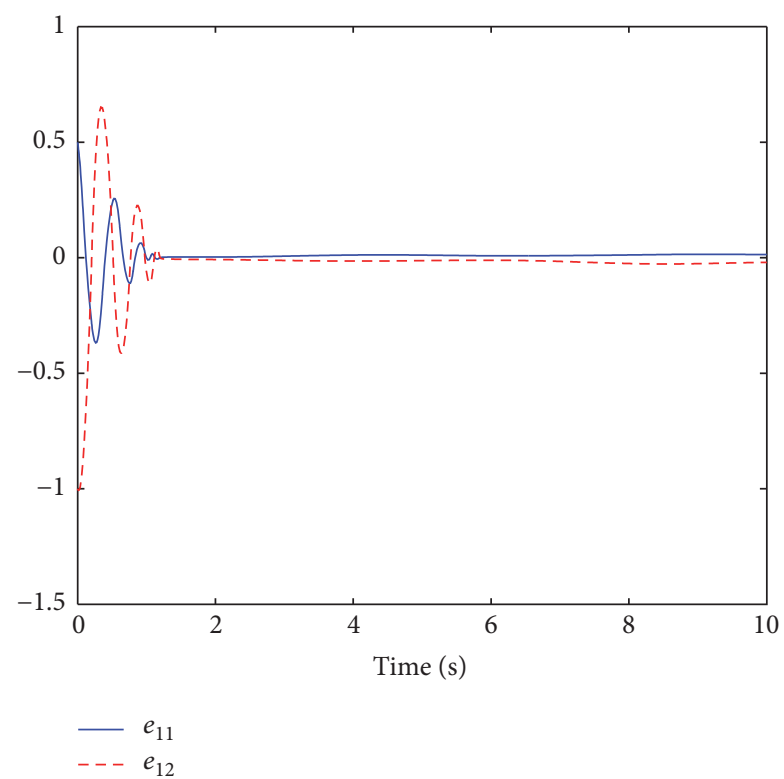

FIgURE 3: Tracking errors to the two desired trajectories $x_{11 d}$ and $x_{12 d}$.

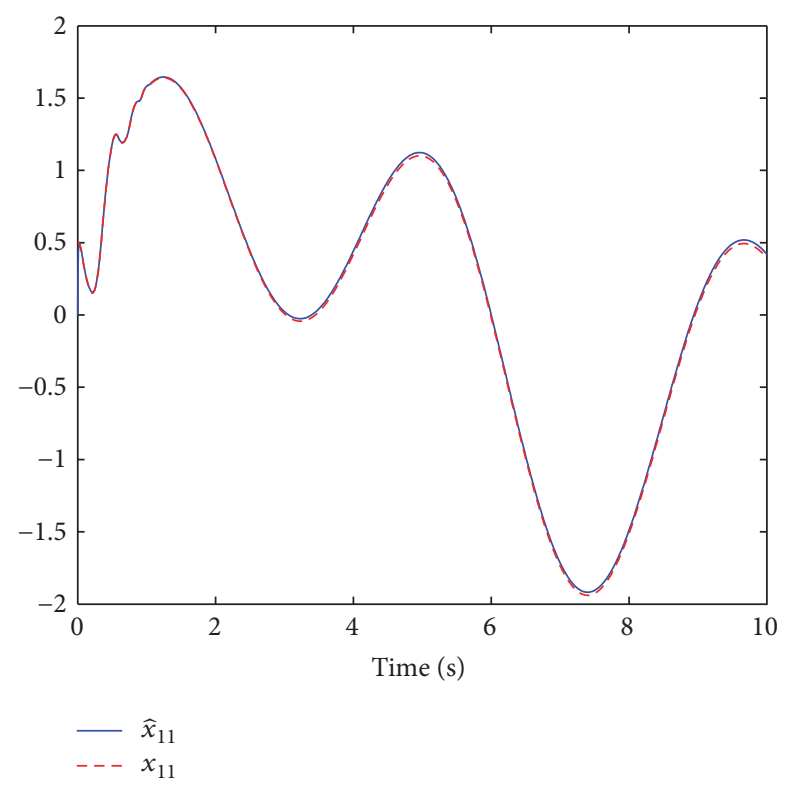

FIgURE 4: Responses of $x_{11}$ and its estimate.

estimate performance is satisfactory and the estimate errors are asymptotically convergent. According to the simulation results presented in Figures 8 and 9, we can also observe that the control input of the developed disturbance observer based adaptive neural output feedback control is bounded and the saturation appears in the initial control stage. However, the saturation phenomenon is ultimately eliminated via the adaptation method under our proposed adaptive output feedback control scheme.

Based on the simulation results of above simulation case, we can conclude that the developed disturbance observer based adaptive neural output feedback control is effective

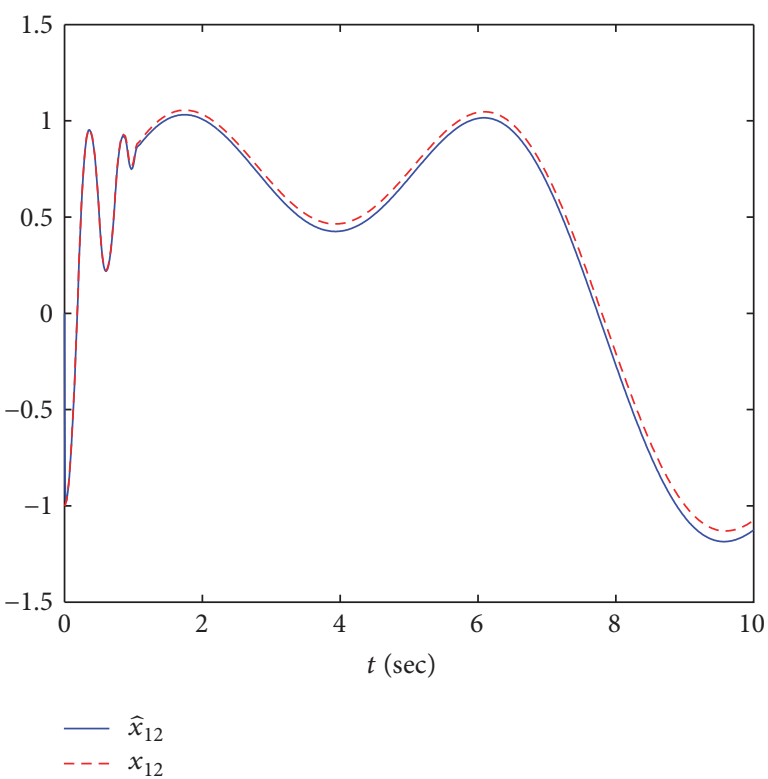

FIgURE 5: Responses of $x_{12}$ and its estimate.

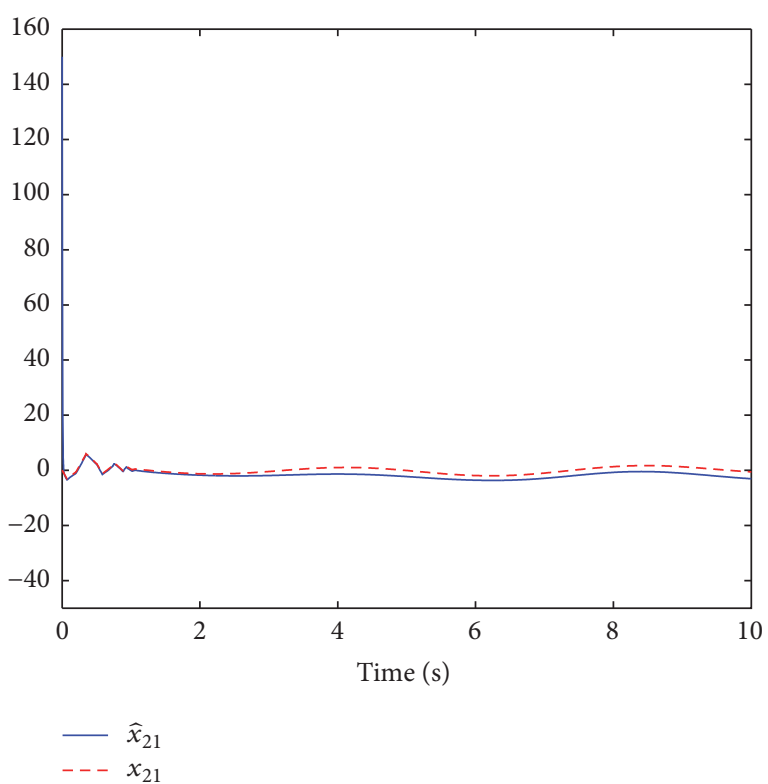

FIgURE 6: Responses of $x_{21}$ and its estimate.

for the two-link robotic manipulator with the time-varying external disturbance, the system uncertainty, and input saturation for the desired bounded time-varying tracking trajectories.

\section{Conclusion}

Disturbance observer based adaptive neural output feedback tracking control has been proposed for the robot manipulator, which is subject to system uncertainty, unknown time-varying external disturbance, and input saturation. The state observer is developed to estimate unmeasured system 


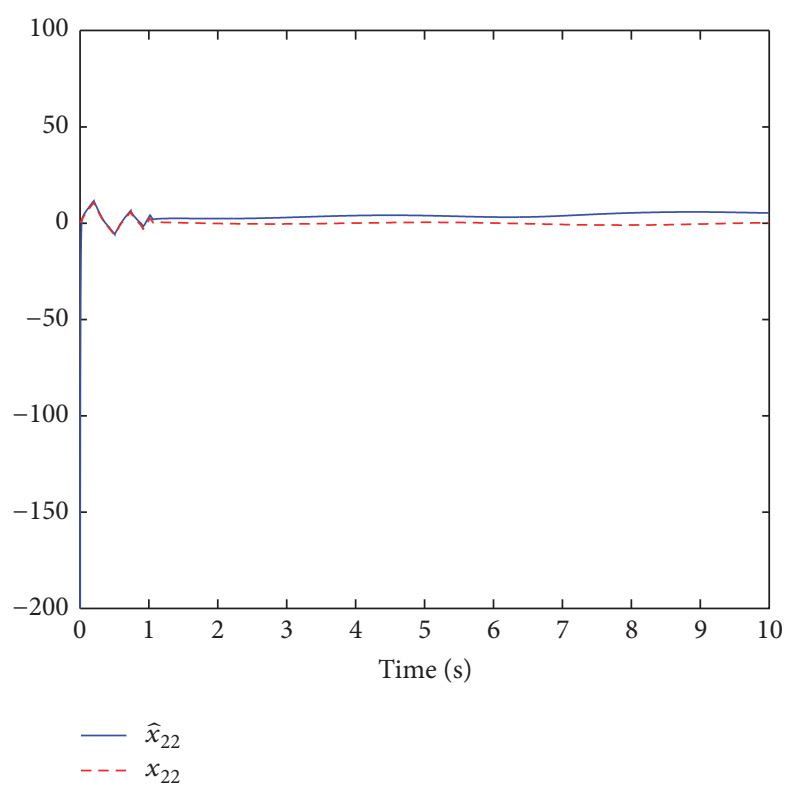

FIGURE 7: Responses of $x_{22}$ and its estimate.

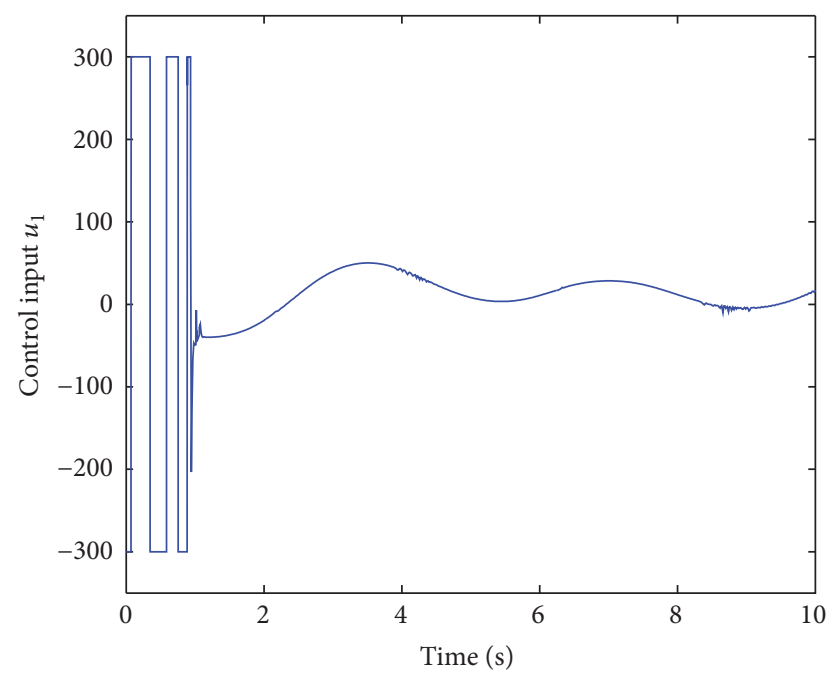

FIGURE 8: Response of the control input $u_{1}$.

states. To improve the ability of disturbance attenuation and the tracking robustness, the disturbance observer has been developed to estimate the combination of the neural network approximation error and the unknown time-varying external disturbance. Based on the outputs of the developed state observer, disturbance observer, and the radial basis function neural network, the adaptive neural output feedback control scheme has been proposed for the robot manipulator. The asymptotical convergence of all closed-loop signals has been proved using rigorous Lyapunov analysis. Simulation results confirm the effectiveness of the proposed disturbance observer based adaptive neural output feedback control scheme.

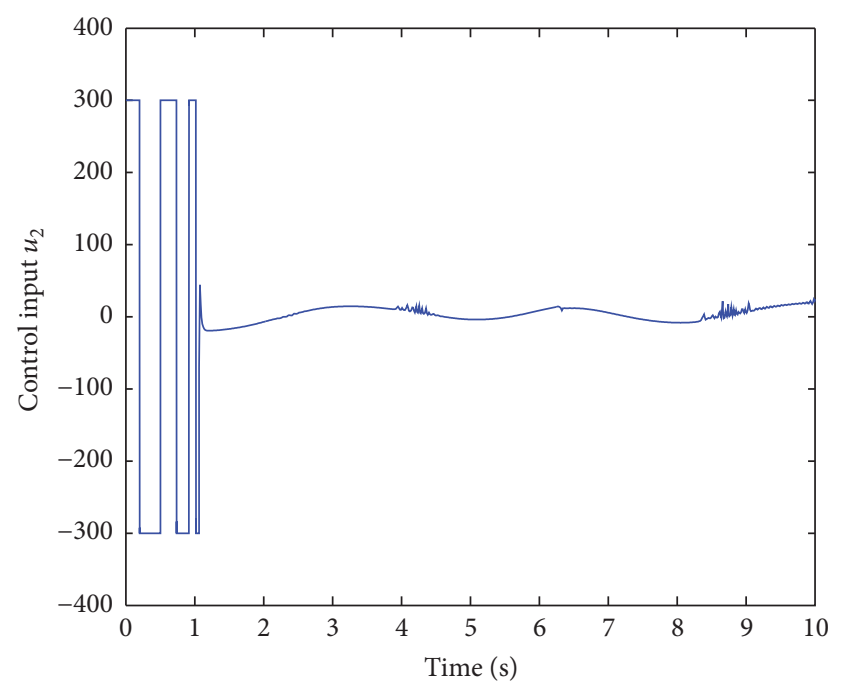

FIGURE 9: Response of the control input $u_{2}$.

\section{Conflicts of Interest}

The authors declare that they have no conflicts of interest.

\section{References}

[1] M. Chen, B. Jiang, and R. X. Cui, "Robust control for rigid robotic manipulators using nonlinear disturbance observer," International Journal of Robotics and Automation, vol. 29, no. 3, pp. 305-311, 2014.

[2] C. Yang, K. Huang, H. Cheng, Y. Li, and C. Su, "Haptic identification by elm-controlled uncertain manipulator," IEEE Transactions on Systems, Man, and Cybernetics: Systems, vol. 47, no. 8, pp. 2398-2409, 2017.

[3] Y. Kang, Z. Li, X. Cao, and D. Zhai, "Robust control of motion/force for robotic manipulators with random time delays," IEEE Transactions on Control Systems Technology, vol. 21, no. 5, pp. 1708-1718, 2013.

[4] J. P. Kolhe, M. Shaheed, T. S. Chandar, and S. E. Talole, "Robust control of robot manipulators based on uncertainty and disturbance estimation," International Journal of Robust and Nonlinear Control, vol. 23, no. 1, pp. 104-122, 2013.

[5] C. Yang, J. Luo, Y. Pan, Z. Liu, and C. Su, "Personalized variable gain control with tremor attenuation for robot teleoperation," IEEE Transactions on Systems, Man, and Cybernetics: Systems, pp. 1-12, 2017.

[6] R.-J. Wai and R. Muthusamy, "Fuzzy-neural-network inherited sliding-mode control for robot manipulator including actuator dynamics," IEEE Transactions on Neural Networks and Learning Systems, vol. 24, no. 2, pp. 274-287, 2013.

[7] S. P. Chan, "A disturbance observer for robot manipulators with application to electronic components assembly," IEEE Transactions on Industrial Electronics, vol. 42, no. 5, pp. 487493, 1995.

[8] J.-J. Slotine and S. S. Sastry, "Tracking control of nonlinear systems using sliding surfaces, with application to robot manipulators," International Journal of Control, vol. 38, no. 2, pp. 465492, 1983.

[9] R.-J. Wai and P.-C. Chen, "Intelligent tracking control for robot manipulator including actuator dynamics via TSK-type fuzzy 
neural network," IEEE Transactions on Fuzzy Systems, vol. 12, no. 4, pp. 552-559, 2004.

[10] W. Dong, "On trajectory and force tracking control of constrained mobile manipulators with parameter uncertainty," Automatica. A Journal of IFAC, the International Federation of Automatic Control, vol. 38, no. 9, pp. 1475-1484, 2002.

[11] Z. Man and M. Palaniswami, "A robust tracking control scheme for rigid robotic manipulators with uncertain dynamics," Computers and Electrical Engineering, vol. 21, no. 3, pp. 211-220, 1995.

[12] L. Cheng, Z.-G. Hou, and M. Tan, "Adaptive neural network tracking control for manipulators with uncertain kinematics, dynamics and actuator model," Automatica, vol. 45, no. 10, pp. 2312-2318, 2009.

[13] M. Chen and G. Tao, "Adaptive fault-tolerant control of uncertain nonlinear large-scale systems with unknown dead zone," IEEE Transactions on Cybernetics, vol. 46, no. 8, pp. 1851-1862, 2016.

[14] J. I. Mulero-Martinez, "Robust GRBF static neurocontroller with switch logic for control of robot manipulators," IEEE Transactions on Neural Networks and Learning Systems, vol. 23, no. 7, pp. 1053-1064, 2012.

[15] M. Chen, G. Tao, and B. Jiang, "Dynamic surface control using neural networks for a class of uncertain nonlinear systems with input saturation," IEEE Transactions on Neural Networks and Learning Systems, vol. 26, no. 9, pp. 2086-2097, 2015.

[16] M. K. Ciliz, "Adaptive control of robot manipulators with neural network based compensation of frictional uncertainties," Robotica, vol. 23, no. 2, pp. 159-167, 2005.

[17] R.-J. Wai, “Tracking control based on neural network strategy for robot manipulator," Neurocomputing, vol. 51, pp. 425-445, 2003.

[18] M. J. Er and Y. Gao, "Robust adaptive control of robot manipulators using generalized fuzzy neural networks," IEEE Transactions on Industrial Electronics, vol. 50, no. 3, pp. 620628, 2003.

[19] R.-J. Wai and P.-C. Chen, "Robust neural-fuzzy-network control for robot manipulator including actuator dynamics," IEEE Transactions on Industrial Electronics, vol. 53, no. 4, pp. 13281349, 2006.

[20] K. W. Lee and H. K. Khalil, "Adaptive output feedback control of robot manipulators using high-gain observer," International Journal of Control, vol. 67, no. 6, pp. 869-886, 1997.

[21] S. C. Tong, T. Wang, Y. M. Li, and B. Chen, "A combined backstepping and stochastic small-gain approach to robust adaptive fuzzy output feedback control," IEEE Transactions on Fuzzy Systems, vol. 21, no. 2, pp. 314-327, 2013.

[22] M. Chen, S. S. Ge, B. V. E. How, and Y. S. Choo, "Robust adaptive position mooring control for marine vessels," IEEE Transactions on Control Systems Technology, vol. 21, no. 2, pp. 395-409, 2013.

[23] Y. Liu, S. Tong, and C. L. P. Chen, "Adaptive fuzzy control via observer design for uncertain nonlinear systems with unmodeled dynamics," IEEE Transactions on Fuzzy Systems, vol. 21, no. 2, pp. 275-288, 2013.

[24] S. Tong, Y. Li, and P. Shi, "Observer-based adaptive fuzzy backstepping output feedback control of uncertain MIMO pure-feedback nonlinear systems," IEEE Transactions on Fuzzy Systems, vol. 20, no. 4, pp. 771-785, 2012.

[25] M. Chen and S. Ge, "Adaptive neural output feedback control of uncertain nonlinear systems with unknown hysteresis using disturbance observer," IEEE Transactions on Industrial Electronics, vol. 26, no. 12, pp. 7706-7716, 2015.
[26] Q. Zhou, P. Shi, S. Xu, and H. Li, "Adaptive output feedback control for nonlinear time-delay systems by fuzzy approximation approach," IEEE Transactions on Fuzzy Systems, vol. 21, no. 2, pp. 301-313, 2013.

[27] F. Alonge, F. D’Ippolito, and F. M. Raimondi, "An adaptive control law for robotic manipulator without velocity feedback," Control Engineering Practice, vol. 11, no. 9, pp. 999-1005, 2003.

[28] Y. H. Kim and F. L. Lewis, "Neural network output feedback control of robot manipulators," IEEE Transactions on Robotics and Automation, vol. 15, no. 2, pp. 301-309, 1999.

[29] W. Zeng and C. Wang, "Learning from NN output feedback control of robot manipulators," Neurocomputing, vol. 125, pp. 172-182, 2014.

[30] P. R. Pagilla and M. Tomizuka, "An adaptive output feedback controller for robot arms: stability and experiments," Automatica. A Journal of IFAC, the International Federation of Automatic Control, vol. 37, no. 7, pp. 983-995, 2001.

[31] M. Chen, "Disturbance attenuation tracking control for wheeled mobile robots with skidding and slipping," IEEE Transactions on Industrial Electronics, vol. 64, no. 4, pp. 33593368, 2017.

[32] M. Chen and B. Jiang, "Robust attitude control of near space vehicles with time-varying disturbances," International Journal of Control, Automation and Systems, vol. 11, no. 1, pp. 182-187, 2013.

[33] M. Chen, B. Ren, Q. Wu, and C. Jiang, "Anti-disturbance control of hypersonic flight vehicles with input saturation using disturbance observer," Science China. Information Sciences, vol. 58, no. 7, 070202, 12 pages, 2015.

[34] M. Chen, P. Shi, and C. C. Lim, "Adaptive neural fault-tolerant control of a 3-dof model helicopter system," IEEE Transactions on Systems, Man, and Cybernetics: Systems, vol. 46, no. 2, pp. 260-270, 2015.

[35] X. Wei and L. Guo, "Composite disturbance-observer-based control and $\mathrm{H} 1$ control for complex continuous models," International Journal of Robust and Nonlinear Control, vol. 20, no. 1, pp. 106-118, 2010.

[36] L. Guo and W.-H. Chen, "Disturbance attenuation and rejection for systems with nonlinearity via DOBC approach," International Journal of Robust and Nonlinear Control, vol. 15, no. 3, pp. 109-125, 2005.

[37] J. Yang, S. Li, and X. Yu, "Sliding-mode control for systems with mismatched uncertainties via a disturbance observer," IEEE Transactions on Industrial Electronics, vol. 60, no. 1, pp. 160-169, 2013.

[38] W.-H. Chen, "Nonlinear disturbance observer-enhanced dynamic inversion control of missiles," Journal of Guidance, Control, and Dynamics, vol. 26, no. 1, pp. 161-166, 2003.

[39] W.-H. Chen, D. J. Ballance, P. J. Gawthrop, and J. O’Reilly, “A nonlinear disturbance observer for robotic manipulators," IEEE Transactions on Industrial Electronics, vol. 47, no. 4, pp. 932-938, 2000.

[40] Z.-J. Yang, Y. Fukushima, and P. Qin, "Decentralized adaptive robust control of robot manipulators using disturbance observers," IEEE Transactions on Control Systems Technology, vol. 20, no. 5, pp. 1357-1365, 2012.

[41] A. Mohammadi, M. Tavakoli, H. J. Marquez, and F. Hashemzadeh, "Nonlinear disturbance observer design for robotic manipulators," Control Engineering Practice, vol. 21, no. 3, pp. 253-267, 2013. 
[42] A. Nikoobin and R. Haghighi, "Lyapunov-based nonlinear disturbance observer for serial n-Link robot manipulators," Journal of Intelligent and Robotic Systems: Theory and Applications, vol. 55, no. 2-3, pp. 135-153, 2009.

[43] M. Chen, P. Shi, and C.-C. Lim, "Robust constrained control for MIMO nonlinear systems based on disturbance observer," Institute of Electrical and Electronics Engineers. Transactions on Automatic Control, vol. 60, no. 12, pp. 3281-3286, 2015.

[44] M. Chen, S. S. Ge, and B. V. E. How, "Robust adaptive neural network control for a class of uncertain MIMO nonlinear systems with input nonlinearities," IEEE Transactions on Neural Networks, vol. 21, no. 5, pp. 796-812, 2010.

[45] M. Chen, S. S. Ge, and B. Ren, "Adaptive tracking control of uncertain MIMO nonlinear systems with input constraints," Automatica, vol. 47, no. 3, pp. 452-465, 2011.

[46] B. Xiao, Q. Hu, and P. Shi, "Attitude stabilization of spacecrafts under actuator saturation and partial loss of control effectiveness," IEEE Transactions on Control Systems Technology, vol. 21, no. 6, pp. 2251-2263, 2013.

[47] M. Chen, "Constrained control allocation for overactuated aircraft using a neurodynamic model," IEEE Transactions on Systems, Man, and Cybernetics: Systems, vol. 46, no. 12, pp. 16301641, 2016.

[48] Y. Li, S. Tong, and T. Li, "Composite adaptive fuzzy output feedback control design for uncertain nonlinear strict-feedback systems with input saturation," IEEE Transactions on Cybernetics, vol. 45, no. 10, pp. 2299-2308, 2015.

[49] M. Chen and S. S. Ge, "Direct adaptive neural control for a class of uncertain nonaffine nonlinear systems based on disturbance observer," IEEE Transactions on Cybernetics, vol. 43, no. 4, pp. 1213-1225, 2013.

[50] C. Yang, Y. Jiang, Z. Li, W. He, and C.-Y. Su, "Neural control of bimanual robots with guaranteed global stability and motion precision," IEEE Transactions on Industrial Informatics, vol. 13, no. 3, pp. 1162-1171, 2017.

[51] M. Chen, B. Jiang, and R. Cui, "Actuator fault-tolerant control of ocean surface vessels with input saturation," International Journal of Robust and Nonlinear Control, vol. 26, no. 3, pp. 542564, 2016.

[52] M. Chen, S. Shao, P. Shi, and Y. Shi, "Disturbance-observerbased robust synchronization control for a class of fractionalorder chaotic systems," IEEE Transactions on Circuits and Systems II: Express Briefs, vol. 64, no. 4, pp. 417-421, 2017.

[53] J. E. Slotine and M. W. Spong, "Robust robot control with bounded input torques," Journal of Robotic Systems, vol. 2, no. 4, pp. 329-352, 1985.

[54] M. W. Spong, J. S. Thorp, and J. M. Kleinwaks, "The control of robot manipulators with bounded input," IEEE Transactions on Automatic Control, vol. 31, no. 6, pp. 483-490, 1986.

[55] H.-S. Choi, "Robust control of robot manipulators with torque saturation using fuzzy logic," Robotica, vol. 19, no. 6, pp. 631-639, 2001.

[56] H. Liu, K. Hao, and X. Lai, "Fuzzy saturated output feedback tracking control for robot manipulators: a singular perturbation theory based approach," International Journal of Advanced Robotic Systems, vol. 8, no. 4, pp. 43-53, 2011.

[57] H. S. Liu, S. Q. Zhu, and Z. W. Chen, "Saturated output feedback tracking control for robot manipulators via fuzzy self-tuning," Journal of Zhejiang University-SCIENCE C, vol. 11, no. 12, pp. 956-966, 2010.
[58] K. S. Eom, I. H. Suh, and W. K. Chung, "Disturbance observer based path tracking control of robot manipulator considering torque saturation," Mechatronics, vol. 11, no. 3, pp. 325-343, 2001.

[59] C.-S. Chiu, K.-Y. Lian, and T.-C. Wu, "Robust adaptive motion/force tracking control design for uncertain constrained robot manipulators," Automatica, vol. 40, no. 12, pp. 2111-2119, 2004.

[60] Y. M. Li, S. C. Tong, and T. S. Li, "Hybrid fuzzy adaptive output feedback control design for uncertain MIMO nonlinear systems with time-varying delays and input saturation," IEEE Transactions on Fuzzy Systems, vol. 24, no. 4, pp. 841-853, 2016.

[61] C.-Y. Su and Y. Stepanenko, "Backstepping-based hybrid adaptive control of robot manipulators incorporating actuator dynamics," International Journal of Adaptive Control and Signal Processing, vol. 11, no. 2, pp. 141-153, 1997.

[62] Q. Hu, L. Xu, and A. Zhang, "Adaptive backstepping trajectory tracking control of robot manipulator," Journal of the Franklin Institute. Engineering and Applied Mathematics, vol. 349, no. 3, pp. 1087-1105, 2012.

[63] M. Zhihong, A. P. Paplinski, and H. R. Wu, "A robust MIMO terminal sliding mode control scheme for rigid robotic manipulators," IEEE Transactions on Automatic Control, vol. 39, no. 12, pp. 2464-2469, 1994.

[64] S. S. Ge and C. Wang, "Adaptive neural control of uncertain MIMO nonlinear systems," IEEE Transactions on Neural Networks, vol. 15, no. 3, pp. 674-692, 2004.

[65] Y. Feng, X. Yu, and Z. Man, "Non-singular terminal sliding mode control of rigid manipulators," Automatica. A, vol. 38, no. 12, pp. 2159-2167, 2002.

[66] K. P. Tee and S. S. Ge, "Control of fully actuated ocean surface vessels using a class of feedforward approximators," IEEE Transactions on Control Systems Technology, vol. 14, no. 4, pp. 750-756, 2006.

[67] S. S. Ge and C. Wang, "Direct adaptive NN control of a class of nonlinear systems," IEEE Transactions on Neural Networks, vol. 13, no. 1, pp. 214-221, 2002.

[68] M. Chen and W.-H. Chen, "Sliding mode control for a class of uncertain nonlinear system based on disturbance observer," International Journal of Adaptive Control and Signal Processing, vol. 24, no. 1, pp. 51-64, 2010. 


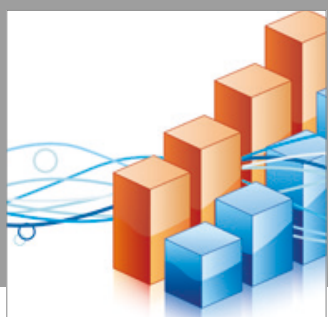

Advances in

Operations Research

vatersals

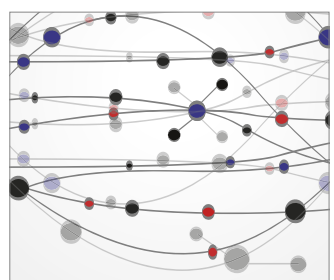

\section{The Scientific} World Journal
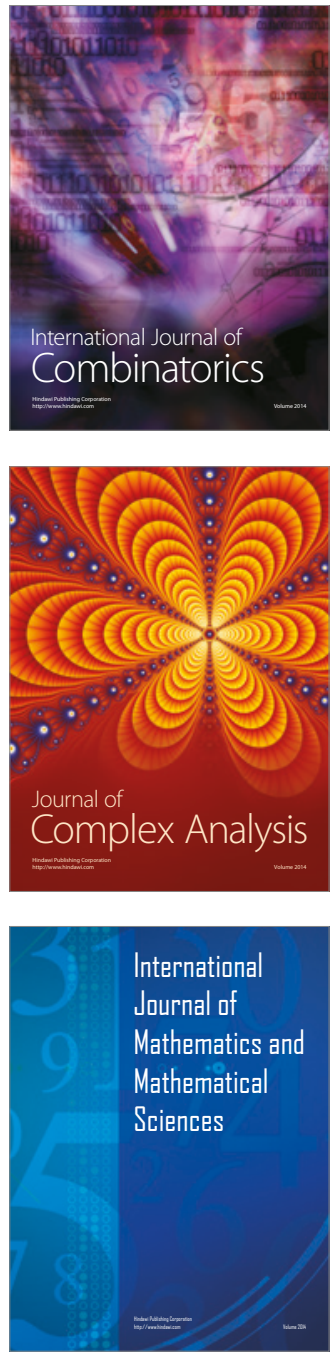
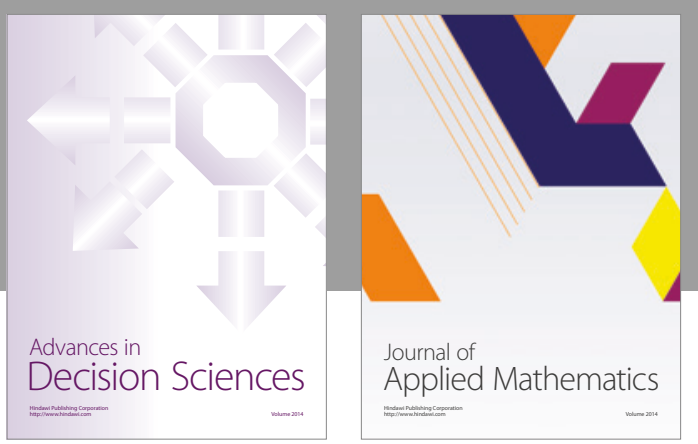

Algebra

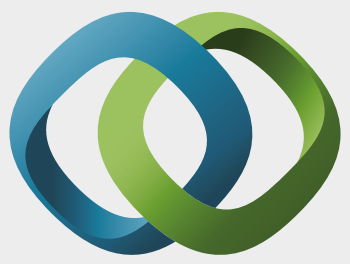

\section{Hindawi}

Submit your manuscripts at

https://www.hindawi.com
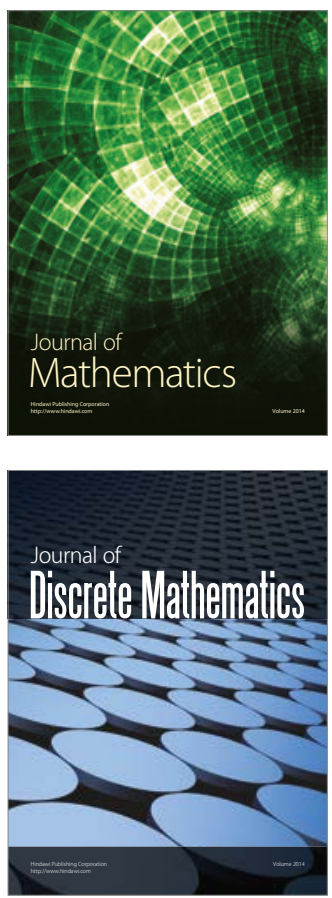

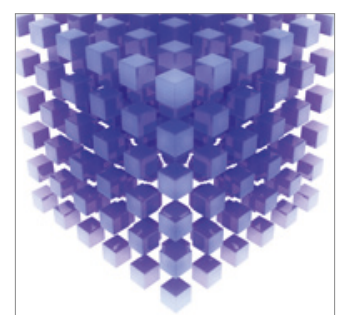

Mathematical Problems in Engineering
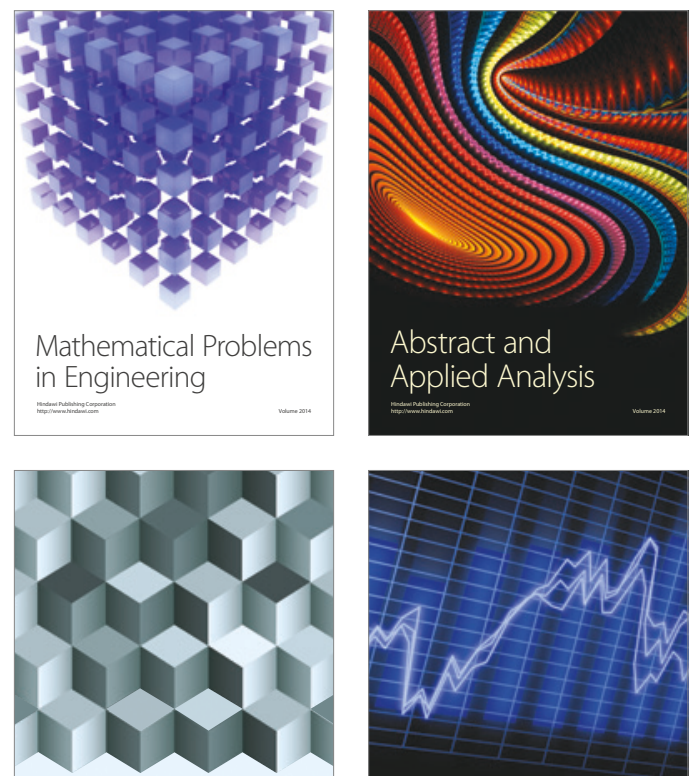

Journal of

Function Spaces

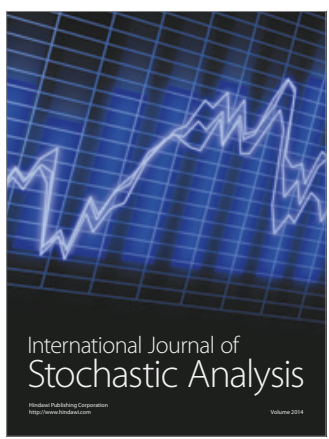

Probability and Statistics
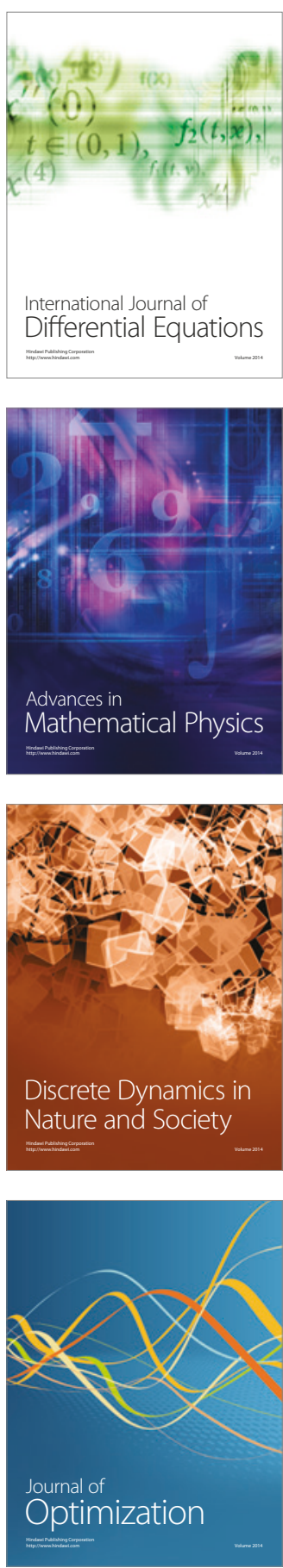